\title{
Automatic 3-D Positioning of Surgical Instruments during Robotized Laparoscopic Surgery Using Automatic Visual Feedback
}

\author{
A. Krupa ${ }^{1}$, M. de Mathelin ${ }^{1}$, C. Doignon ${ }^{1}$, J. Gangloff $^{1}$, G. Morel $^{\star}$, \\ L. Soler ${ }^{2}$, J. Leroy ${ }^{2}$, and J. Marescaux ${ }^{2}$ \\ 1 LSIIT (UMR CNRS 7005), University of Strasbourg I, France \\ \{alexandre.krupa, michel.demathelin\}@ensps.u-strasbg.fr \\ 2 IRCAD (Institut de Recherche sur le Cancer de l'Appareil Digestif), France
}

\begin{abstract}
This paper presents a robotic vision system that automatically retrieves and positions surgical instruments in the robotized laparoscopic surgical environment. The goal of the automated task is to bring the instrument from an unknown or hidden position to a desired location specified by the surgeon on the endoscopic image. To achieve this task, a special instrument-holder is designed that projects laser dot patterns onto the organ surface which are seen on the endoscopic images. Then, the surgical instrument positioning is done using an automatic visual servoing from the endoscopic image. Our approach is successfully validated in a real surgical environment by performing experiments on living pigs in the surgical training room of IRCAD.
\end{abstract}

\section{Introduction}

Robotic laparoscopic systems have recently appeared, e.g., ZEUS (Computer Motion, Inc.) or DaVinci (Intuitive Surgical, Inc.). With these systems, robot arms are used to manipulate the surgical instruments as well as the endoscope. The surgeon tele-operates the robot through master arms using the visual feedback from the laparoscope. This reduces the surgeon tiredness, and potentially increases motion accuracy by the use of a high master/slave motion ratio. Furthermore, teleoperation allows to perform long distance surgical procedures (see e.g. [1]). In a typical surgical procedure, the surgeon first drives the laparoscope into a region of interest (for example by voice, with the AESOP system of Computer Motion Inc.). Then, he or she drives the surgical instruments at the operating position. A practical difficulty lies in the fact that the instruments are generally not in the field of view at the start of the procedure. Therefore, the surgeon must either blindly move the instruments into the field of view of the endoscope, or zoom out with the endoscope as to get a larger view. Similarly, when the surgeon zoom in or moves the endoscope during surgery, the instruments may leave the endoscope's field of view. Consequently, instruments

^ Now with the University of Paris 6 - LRP6 - Paris - France

T. Dohi and R. Kikinis (Eds.): MICCAI 2002, LNCS 2488, pp. 9-16 2002.

(C) Springer-Verlag Berlin Heidelberg 2002 
may have to be moved blindly with a risk of an undesirable contact between instruments and internal organs.

Therefore, in order to assist the surgeon, we propose a visual servoing system that automatically brings the instrument at the center of the endoscopic image (see, 2 2] and 3] for earlier works in that direction). Furthermore, when the instrument is in the field of view, the system provides a measurement of the distance between the organ and the tip of the instrument. Our system also allows to move automatically the instruments at a 3-D location chosen by the surgeon. The surgeon specifies the position in the image (with, e.g., a touch-screen or a mouse-type device) and sets the distance value between the instrument and the organ at his or her convenience. Note that prior research was conducted on visual servoing techniques in laparoscopic surgery in, e.g., [4], 5] and [6]. However, these systems were guiding the endoscope toward the region of interest instead of the instruments.

Our system includes a special device designed to hold the surgical instruments with tiny laser pointers. This laser pointing instrument-holder is used to project laser spots in the laparoscopic image even if the surgical instrument is not in the field of view. The image of the projected laser spots is used to automatically guide the instrument in the field of view. A major difficulty in designing this automatic instrument positioning system lies in the unknown relative position between the endoscope and the robot holding the instrument, and in the use of monocular vision that lacks depth information. In [7], the authors added an extra laser-pointing endoscope with an optical galvano scanner and a $955 \mathrm{fps}$ high-speed camera to estimate the 3 -D surface of the scanned organ. However, their method requires to know the relative position between the laser-pointing endoscope and the camera which is estimated by adding an external camera watching the whole system (OPTOTRAK system). In our approach, instead, we robustly estimate in real time the distance between instrument and organ by using images of optical markers mounted on the tip of the instrument and images of the laser spots projected by the same instrument. Then, visual feedback allows to automatically position the instrument at a desired location.

The paper is organized as follows. Section 2 describes the system configuration and the endoscopic laser pointing instrument prototype. In section 3, robust image processings leading to laser patterns and optical markers detection are briefly explained. The control scheme used to position the instrument by automatic visual feedback is described in section 4 . In the final section, we present experimental results in real surgical conditions at the operating room of IRCAD.

\section{System Description}

\subsection{System Configuration}

The system configuration used to perform the autonomous positioning of the surgical instrument is shown in figure 1,a. The system includes a laparoscopic surgical robot and an endoscopic laser pointing instrument-holder. The robot allows to move the instrument across a trocar placed at a first incision point. A 

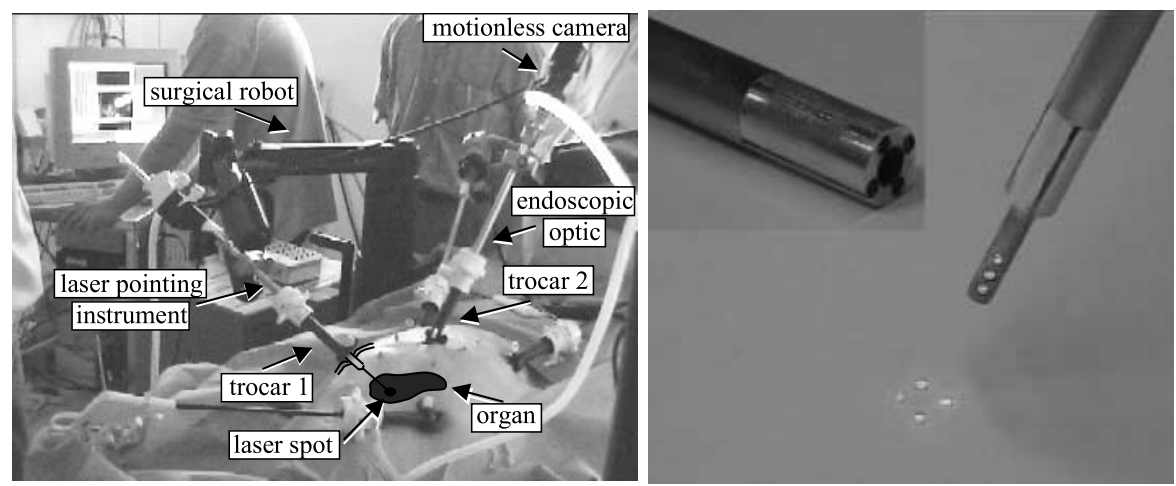

Fig. 1. (a) System configuration (b)-(c) Endoscopic laser pointing instrument-holder

second trocar is put in order to insert an endoscopic optical lens which provides the feedback view and whose relative position to the robot base frame is generally unknown.

\subsection{Endoscopic Laser Pointing Instrument-Holder}

We have designed the prototype of an endoscopic laser pointing instrumentholder shown in figure 1.b. It is a $30 \mathrm{~cm}$ long metallic pipe with $10 \mathrm{~mm}$ external diameter to be inserted through a $12 \mathrm{~mm}$ standard trocar. Its internal diameter is $5 \mathrm{~mm}$ so that a standard laparoscopic surgical instrument can fit inside. The head of the instrument-holder contains miniature laser collimators connected to optical fibers which are linked to external controlled laser sources. This device allows to remote laser sources which can not be integrated in the head of the instrument due to their size. The laser pointing instrument-holder mounted on a cleaning-suction instrument is shown in figure 1.c. The instrument-holder, with the surgical instrument inside, is held by the end-effector of the robot. It allows to project optical markers on the surgical scene. Three tiny light emitting diodes are fixed on the tip of the instrument to provide the optical markers needed to compute the depth information.

\section{Robust Detection of Lasers Spots and Optical Markers}

Robust detection of the geometric center of the projected laser spots in the image plane and detection of the image positions of the optical markers mounted on the instrument are needed in our system. Due to the complexity of the organ surface, laser spots may be occulted. Therefore a high redundancy factor is achieved by using at least four laser pointers. We have found in our experiments in vivo with four laser sources that the computation of the geometric center is always possible with a small bias even if three spots are occulted. Due to the viscosity of the organs, the surfaces are bright and laser spots are diffused. Therefore, the main difficulty is to precisely detect diffused laser points from 
the images speckle due to the ligthing conditions. A simple image binarization is not suitable. Our approach consists in synchronizing the laser sources with the image capture board in such a way that the laser spots are turned on during the even field of image frames and are turned off during the odd frames. In this manner, laser spots are visible one line out of two and can be robustly detected with a highpass spatial 2D FIR filter. Figure 2 shows images resulting
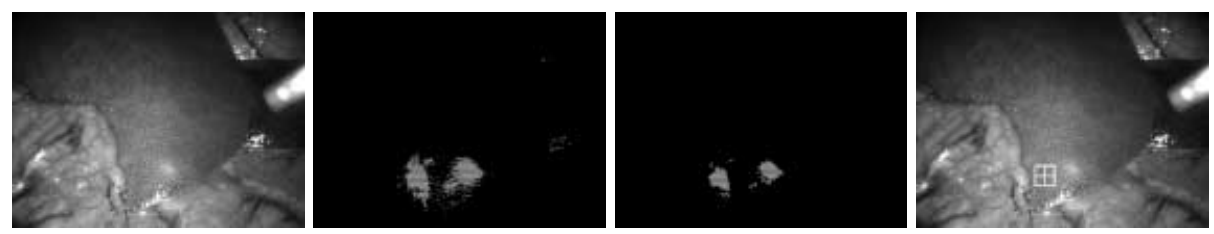

Fig. 2. Robust laser spot detection on a liver

from different steps of the image processing (original image - hightpass filtering thresholding and erosion - detected geometric center). Coordinates of the center of mass are then computed from the filtered image. Note that the motions of organs due to heart beat and breathing may be also extracted with this spatial filtering of the image interlacing. They act like noise on the computed geometric center. Therefore a lowpass time filtering is also applied on the center of mass coordinates to reduce this noise. The optical markers mounted on the instrument are also synchronized with the image capture board. In contrast with the laser sources, the three optical markers are turned on during the odd frames and turned off during the even frames. Using the same method of detection, the image of optical markers is extracted. Coordinates of the center of the markers are then obtained using an ellipse detection algorithm (see figure 3, a).

\section{Instrument Positioning with Visual Servoing}

In laparoscopic surgery, instrument displacements are constrained to 4 DOF through an incision point. Only three rotations and one translation are allowed. Let $R_{L}$ be a frame of reference at the tip of the instrument and $R_{C}$ the camera frame (see figure 3.b). We define the instrument's velocity vector $\dot{W}=\left(\begin{array}{llll}\omega_{x} & \omega_{y} & \omega_{z} & v_{z}\end{array}\right)^{T}$ where $\omega_{x}, \omega_{y}$ and $\omega_{z}$ are rotational velocities of frame $R_{L}$ with respect to camera frame $R_{C}$ expressed in $R_{L}$ and $v_{z}$ is the translational velocity of the instrument along the $z_{L}$ axis. In the case of a symmetrical instrument like the cleaning-suction instrument, it is not necessary to turn the instrument around its own axis to do the desired task. So only 3 DOF will be actuated for this kind of instrument and the operational space velocity vector is reduced to $\dot{W}_{o p}=\left(\begin{array}{lll}\omega_{x} & \omega_{y} & v_{z}\end{array}\right)^{T}$. For a non symmetrical instrument like a clamping instrument, the rotational speed $\omega_{z}$ can be kept in vector $\dot{W}_{o p}$. To perform a positioning of the instrument, the surgeon specifies the target point on the screen. The required visual features are image coordinates $S_{p}=\left(\begin{array}{ll}u_{p} & v_{p}\end{array}\right)^{T}$ 


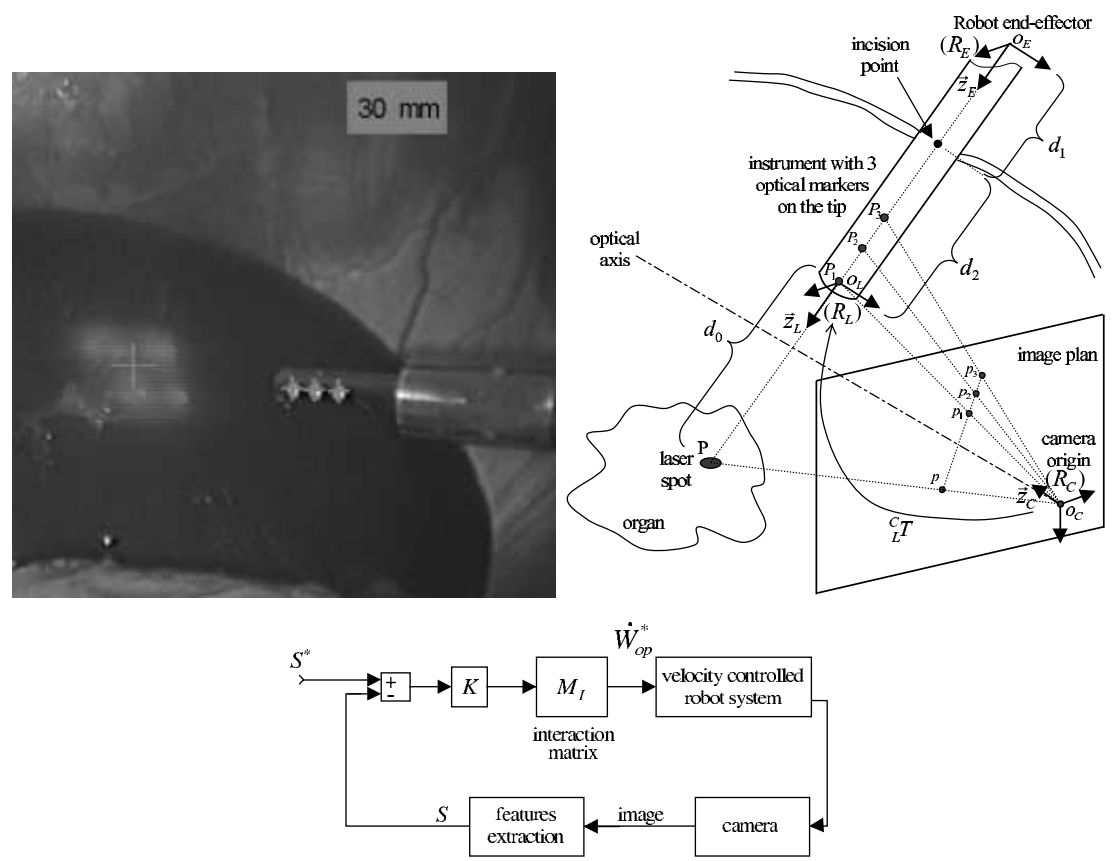

Fig. 3. (a) Robust optical markers detection (b) System geometry (c) Control scheme

of the center of mass of the projected laser spots. This center of mass is seen as the projection of the instrument axis onto the organ. Adding optical markers on the tip of the instrument allows to compute the distance $d_{0}$ between the organ and the instrument (see section 4.1). Figure 3.c shows the control scheme used for this positioning. The feature vector to control is $S=\left(\begin{array}{lll}u_{p} & v_{p} & d_{0}\end{array}\right)^{T}$. If the classical image-based control strategy is used for visual servoing (cf. [8]), the reference velocity vector $\dot{W}_{o p}^{*}$ is chosen proportional to the error on the features: $\dot{W}_{o p}^{*}=M_{I} K\left(S^{*}-S\right)$ where $K$ is a positive diagonal gain matrix, $M_{I}$ is the interaction matrix, $M_{I}=J_{S}^{-1}$ in classical visual servoing where $J_{S}$ is a Jacobian matrix relating the instrument velocity vector $\dot{W}_{o p}$ to the image feature velocity vector $\dot{S},\left(\dot{S}=J_{S} \dot{W}_{o p}\right)$. Furthermore, $S^{*}=\left(\begin{array}{ll}S_{p}^{*} & d_{0}^{*}\end{array}\right)^{T}$, where $S_{p}^{*}=\left(\begin{array}{ll}u_{p}^{*} & v_{p}^{*}\end{array}\right)^{T}$ is the desired instrument position in the image and $d_{0}^{*}$ is the desired distance between the organ and the tip of the instrument. The distance between organ and instrument velocity can be measured only if the laser spots and the instrument markers are visible (see section 4.1). When the tip of the instrument is not in the field of view, only $S_{p}$ can be controlled. Therefore, the instrument retrieval and positioning algorithm must have three stages:

Stage 1: Positioning of the laser spot at the center of the image by visual servoing of $S_{p}$ only; control of $\left(\begin{array}{ll}\omega_{x} & \omega_{y}\end{array}\right)^{T}$ only, with $\dot{W}_{o p}^{*}=\left(\begin{array}{lll}\omega_{x}^{*} & \omega_{y}^{*} & 0\end{array}\right)^{T}$.

Stage 2: Bringing down the instrument along its axis until the optical markers are in the field of view; open-loop motion at constant speed $v_{z}^{*}$ with $\dot{W}_{o p}^{*}=$ $\left(\begin{array}{lll}0 & 0 & v_{z}^{*}\end{array}\right)^{T}$. 
Stage 3: Full visual servoing.

\subsection{Depth Measurement}

Adding optical markers on the tip of the instrument allows to measure the distance $d_{0}$. We put 3 markers $P_{1}, P_{2}, P_{3}$ along the instrument axis in such a way that we can assume that $P_{1}, P_{2}, P_{3}$ are collinear with the center of mass of the laser spots $P$ (see Figure [3.a). Then, the projective cross-ratio $\tau$ (cf.[9]) is computed from points $P, P_{1}, P_{2}, P_{3}$ and their respective projection in order to estimate the depth $d_{0}$ :

$$
\tau=\frac{\left(\frac{\overline{p p_{2}}}{\overline{p_{1} p_{2}}}\right)}{\left(\frac{\overline{p p_{3}}}{\overline{p_{1} p_{3}}}\right)}=\frac{\left(\overline{\frac{P P_{2}}{P_{1} P_{2}}}\right)}{\left(\frac{\overline{P P_{3}}}{\overline{P_{1} P_{3}}}\right)} \text {. and } \quad d_{0}=\overline{P P_{1}}=(\tau-1) \frac{\overline{P_{1} P_{2}} \cdot \overline{P_{1} P_{3}}}{\overline{P_{1} P_{3}}-\tau \cdot \overline{P_{1} P_{2}}} .
$$

\subsection{Interaction Matrix}

To control the position of the instrument in the image plane, one needs to compute the Jacobian matrix $J_{S}$ which relates the visual features velocity $\dot{S}$ and the instrument velocity $\dot{W}_{o p}$. This Jacobian matrix can be computed analytically if the camera is motionless and if the relative position of the instrument with respect to the camera can be measured as well as the depth variation (if the instrument is in the field of view). For safety reasons, it is not suitable that the laser spots centering error signals, $\left(S_{p}^{*}-S_{p}\right)$, induces depth motions $\left(v_{z}^{*} \neq 0\right)$. Furthermore, when the instrument is going up or down $\left(v_{z}^{*} \neq 0\right)$, this motion does not induce an error on the laser spot centering. Therefore, it is recommended to choose the interaction matrix $M_{I}$ as follows:

$$
M_{I}=\left(\begin{array}{cc}
A_{[2 \times 2]} & 0_{[2 \times 1]} \\
0_{[1 \times 2]} & -1
\end{array}\right) . \quad \widehat{A}=\left(\begin{array}{cc}
\frac{\Delta u_{p}}{\omega_{x}^{*} \Delta T} & \frac{\Delta u_{p}}{\omega_{y}^{*} \Delta T} \\
\frac{\Delta v_{p}}{\omega_{x}^{*} \Delta T} & \frac{\Delta v_{p}}{\omega_{y}^{*} \Delta T}
\end{array}\right)^{-1} .
$$

The matrix $A$ in (2) can be analytically computed from $J_{S}$ if the instrument is visible in the image. When it is not the case, as is stage 1 of the algorithm, this matrix is identified in an initial procedure. This initial identification procedure consists in applying a constant velocity reference signal $\dot{W}_{o p}^{*}=\left(\begin{array}{lll}\omega_{x}^{*} & \omega_{y}^{*} & 0\end{array}\right)^{T}$ during a short time interval $\Delta T$. Small variations of laser spot image coordinates are measured and the estimation of $A$ is given by $\widehat{A}$ in (2). The stability of the visual feedback loop is always guaranteed as long as $A \widehat{A}^{-1}$ remains positive definite [10. Should the laser spot centering performance degrades, it is always possible to start again the identification procedure.

\section{Experiments}

Experiments in real surgical conditions on living pigs were conducted in the operating room of IRCAD (see Figure 1]a). The experimental surgical robotic 
task was the autonomous retrieval of a instrument not seen in the image and then its positioning at a desired 3-D position. We use a bi-processor PC computer $(1.7 \mathrm{GHz})$ for image processing and for controlling, via a serial link, the surgical robot from ComputerMotion. A standard $50 \mathrm{fps}$ PAL endoscopic camera hold by a second robot (at standstill) is linked to a PCI image capture board that grabs grey-level images of the observed scene. For each image the center of mass of the laser spots is detected in about $20 \mathrm{~ms}$. The successive steps in the autonomous retrieval and positioning procedure are as following:

Step 1: changing the orientation of the instrument by applying rotational velocity trajectories (on $\omega_{x}^{*}$ and $\omega_{y}^{*}$ ) in open loop to scan the organ surface with the laser spot until they appear in the endoscopic view.

Step 2: automatic identification of the interaction matrix $A$ (cf.(2)).

Step 3: centering of the laser spots in the image by visual servoing.

Step 4: descent of the instrument by applying a velocity reference signal $v_{z}^{*}$ in open loop until it appears in the image, keeping orientation servoing.

Step 5: real time estimation of the distance $d_{0}$ and depth servoing to reach the desired distance, keeping orientation servoing.

Step 6: new positioning of the instrument to desired 3-D locations by automatic visual servoing at the command of the surgeon. The surgeon indicates on the screen the new laser point image coordinates, $S_{p}^{*}=\left(\begin{array}{ll}u_{p}^{*} & v_{p}^{*}\end{array}\right)^{T}$, and specifies the new desired distance $d_{0}^{*}$ to be reached. Then the visual servoing algorithm performs the positioning.

Figure 4.a-d shows experimental measurements of the laser image coordinates $u_{p}$ and $v_{p}$ during the identification stage of $A$ and during the centering stage by visual servoing. For the identification procedure, four positions have been considered to relate variations of the laser image position and angular variations. We can notice a significant perturbation due to the breathing during the visual servoing. For robust identification purpose, we average several measurements of small displacements. This allows to reduce the effect of the breathing which acts like a disturbance. Figure 4 e shows the 2D trajectory obtained (in the image)
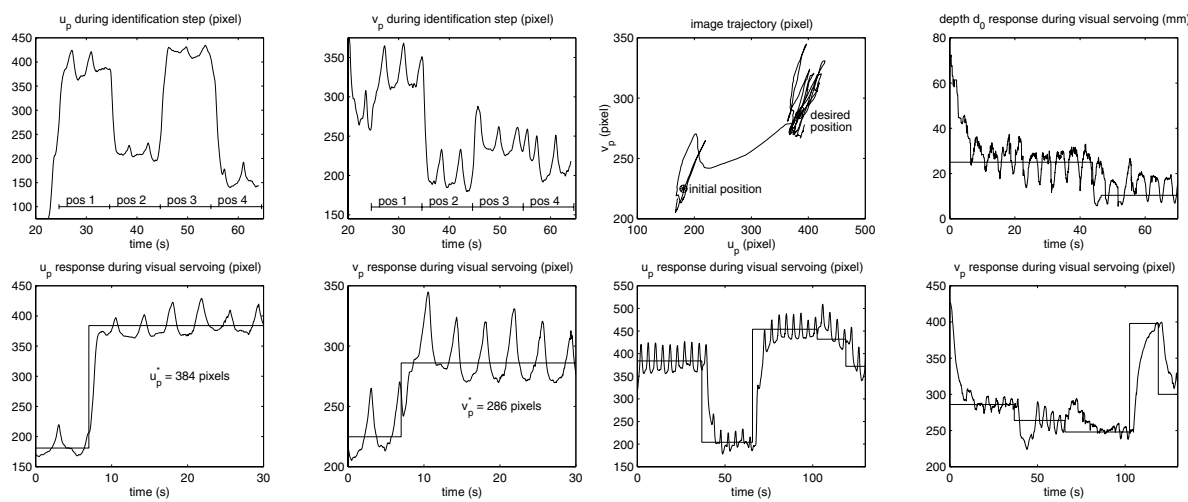

Fig. 4. (a)(b)(c)(d)-(e)(f)(g)(h) Experimental measurements 
during the centering step by visual servoing. The oscillating motion around the initial and desired position are also due to the effect of breathing that acts as a periodic perturbation. Figure 4 f shows the measured distance $d_{0}$ during the depth servoing at step 5. Responses $4 \mathrm{~g}$ and $4 \mathrm{~h}$ display the laser image coordinates when the surgeon specifies new positions to be reached in the image.

\section{Acknowledgements}

The financial support of the french ministry of research ("ACI jeunes chercheurs" program) is gratefully acknowledged. The experimental part of this work has been made possible thanks to the collaboration of Computer Motion Inc. that has graciously provided the medical robots. In particular, we would like to thank Dr. Moji Ghodoussi for his technical support in the use of the medical robots.

\section{References}

1. J. Marescaux, J. Leroy, M. Gagner, and al. Transatlantic robot-assisted telesurgery. Nature, 413:379-380, 2001.

2. A. Krupa, C. Doignon, J. Gangloff, M. de Mathelin, L. Soler, and G. Morel. Towards semi-autonomy in laparoscopic surgery through vision and force feedback control. In Proc. of the Seventh International Symposium on Experimental Robotics (ISER), Hawaii, December 2000.

3. A. Krupa, M. de Mathelin, C. Doignon, J. Gangloff, G. Morel, L. Soler, and J. Marescaux. Development of semi-autonomous control modes in laparoscopic surgery using visual servoing. In Proc. of the Fourth International Conference on Medical Image Computing and Computer-Assisted Intervention (MICCAI), pages 1306-1307, Utrecht, The Netherlands, October 2001.

4. G.-Q. Wei, K. Arbter, and G. Hirzinger. Real-time visual servoing for laparoscopic surgery. IEEE Engineering in Medicine and Biology, 16(1):40-45, 1997.

5. G.-Q. Wei, K. Arbter, and G. Hirzinger. Automatic tracking of laparoscopic instruments by color-coding. In Springer Verlag, editor, Proc. First Int. Joint Conf. CRVMed-MRCAS'97, pages 357-366, Grenoble, France, March 1997.

6. A. Casals, J. Amat, D. Prats, and E. Laporte. Vision guided robotic system for laparoscopic surgery. In Proc. of the IFAC International Congress on Advanced Robotics, pages 33-36, Barcelona, Spain, 1995.

7. M. Hayashibe and Y. Nakamura. Laser-pointing endoscope system for intraoperative 3Dgeometric registration. In Proc. of the 2001 IEEE International Conference on Robotics and Automation, Seoul, Korea, May 2001.

8. F. Chaumette, P. Rives, and B. Espiau. Classification and realization of the different vision-based tasks. In World Scientific Series in Robotics and Automated Systems, Vol. 7, pages 199-228. Visual Servoing, K. Hashimoto (ed.), Singapore, 1993.

9. S.J. Maybank. The cross-ratio and the J-invariant. Geometric invariance in computer vision, Joseph L. Mundy and Andrew Zisserman, MIT press:107-109, 1992.

10. B. Espiau, F. Chaumette, and P. Rives. A new approach to visual servoing in robotics. IEEE Trans. on Robotics and Automation, 8(3):313-326, june 1992. 